\title{
Multiple joint tuberculosis presenting as HLA-B27 disease
}

\author{
G. O. HOPKINS* \\ M.B., M.R.C.P. \\ Department of Medicine, Cambridge Military Hospital, Aldershot, Hampshire
}

\begin{abstract}
Summary
A case of multifocal osteoarticular tuberculosis in a young Caucasian male is presented. The diagnostic difficulty, compounded by slow progression of the disorder and the presence of the tissue antigen HLAB27, is discussed.
\end{abstract}

KEY WORDS: osteoarticular tuberculosis, HLA-B27 antigen, Reiter's disease, ankylosing spondylitis.

\section{Introduction}

The seronegative spondarthritides, ankylosing spondylitis and Reiter's disease have a prevalence in young men of the order of $1 \%$ (Lawrence, 1977). Xray changes, however, may not appear for some time after the onset of clinical symptoms giving rise to a diagnostic delay of some years. Detection of the tissue antigen HLA-B27 is useful in increasing the degree of suspicion in early cases (Brewerton et al., 1973a, 1973b) but it is not diagnostic. Its presence may, as in the present case, be misleading.

\section{Case report}

A 19-year-old Caucasian army recruit presented with a swollen tender left knee and mild backache at his preliminary medical examination. He was seen by a specialist in his home town, where a diagnosis of an HLA-B27 positive, sero-negative arthritis was made and hydrocortisone injected into the joint with complete resolution.

Five months later at basic training, his left knee once again became painful with a large warm effusion. He was apyrexial, and X-ray of the joint was normal. One-hundred millilitres of slightly cloudy, watery, yellow fluid was aspirated, microscopy showing a white cell count of $4.8 \times 10^{9} /$ litre and a few red blood cells, but no bacteria or crystals. Culture, subculture and tests for rheumatoid factor were negative. He had a mild normocytic, normo-

\footnotetext{
* Present address: Department of Rheumatology, The Middlesex Hospital, London W1P 9PG.
}

chromic anaemia, a normal white blood count and an erythrocyte sedimentation of $78 \mathrm{~mm} / \mathrm{hr}$. Hydrocortisone acetate was again injected into the joint.

Two days after admission the patient ran a low grade evening pyrexia and complained of low back pain. Examination revealed a cool, non-tender fluctuant swelling overlying his 5 th lumbar vertebra and extending into the right buttock. $\mathrm{X}$-rays showed loss of definition of the anterior margin of the fifth lumbar vertebra (Fig. 1). Penetrated chest X-ray (Fig. 2) revealed a large para-vertebral abscess and lateral tomography showed anterior erosion of the bodies of vertebrae D6-10.

The presumptive diagnosis was of a tuberculous infection and treatment with streptomycin, rifampicin, isoniazid and pyridoxine was started. A week later his lumbar spine was explored. Necrotic material was removed from the anterior part of L5 with bone chips, and $2 \mathrm{~g}$ of streptomycin powder were packed into the cavity. Aspirate from the lumbar abscess was positive for Mycobacterium tuberculosis on fluorescent stain, culture and guinea-pig inoculation and the organism was found to be sensitive to all 3 drugs. The left knee aspirate, however, was negative on direct staining but positive on culture. Histological examination of bone curettings, and synovial tissue from his knee showed granulomata consistent with tuberculous osteomyelitis. Postoperatively, he had a mild left 5th lumbar root lesion.

Following bedrest for 6 weeks, he was gradually mobilized in a thoraco-lumbar corset. At 8 months he was completely asymptomatic. X-rays showed considerable diminution of the thoracic paravertebral abscess and resolution of the lumbar lesion.

\section{Discussion}

Reports of multifocal osteoarticular tuberculosis are uncommon. Out of 132 patients based in the United Kingdom recently reported (Newton, Sharp and Barnes, 1982; Halsey, Reeback and Barnes, 1982) only 8, all immigrants, had 2 simultaneous sites of skeletal infection and no cases were reported of a 


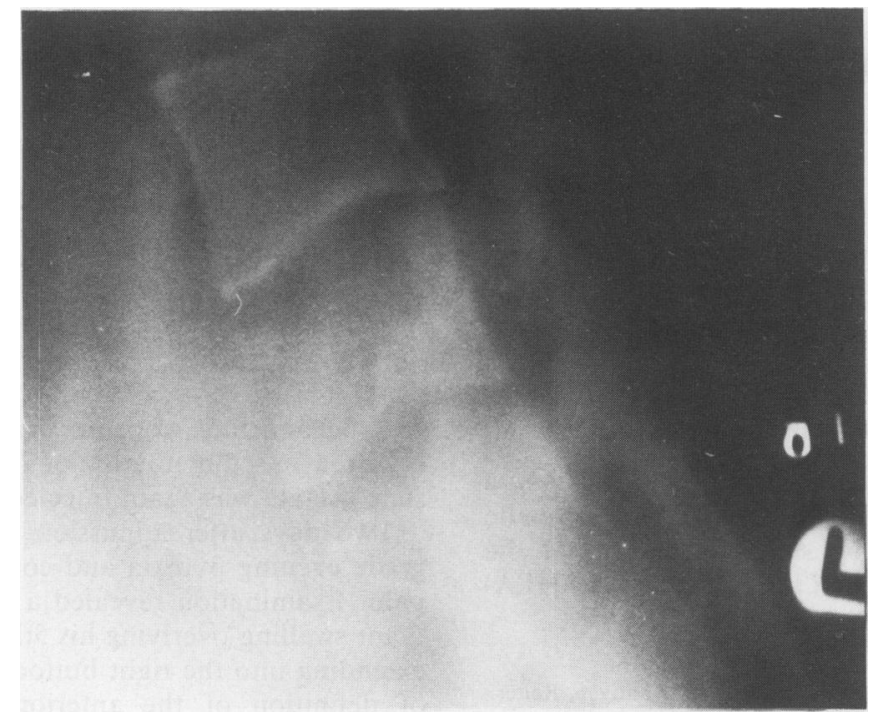

FIG. 1. Lateral lumbar-sacral tomogram showing destruction of 5th lumbar vertebra.

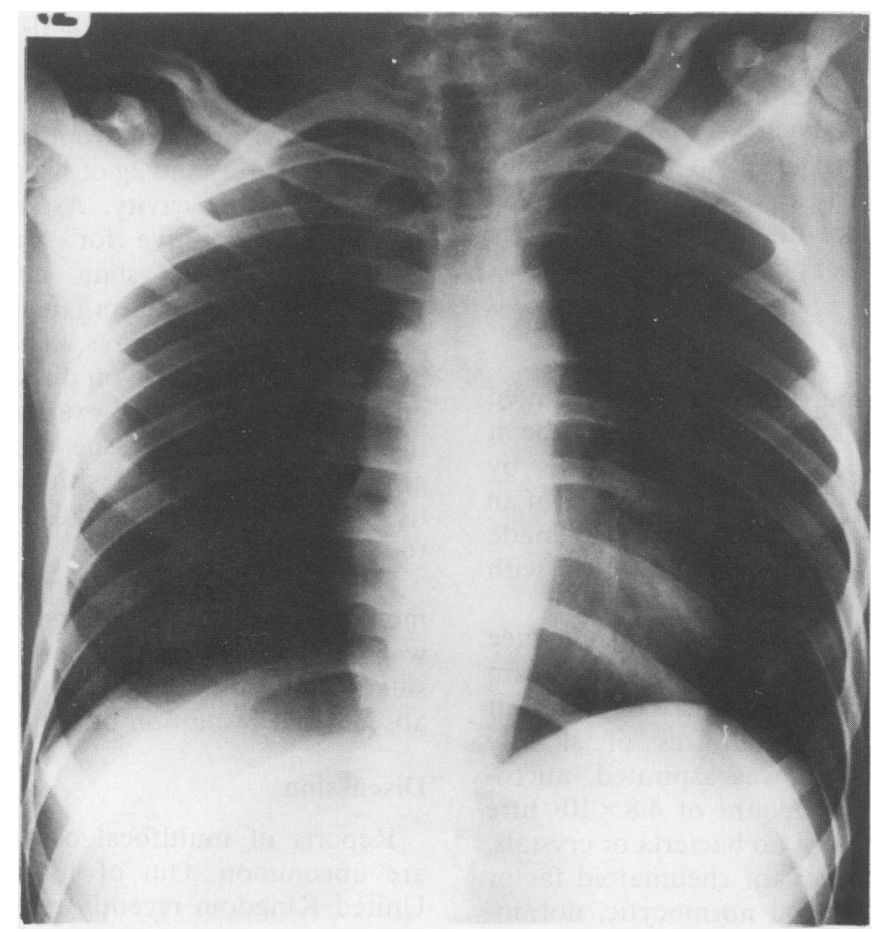


more widespread involvement. The likelihood of multiple lesions occurring in a Caucasion in this country is therefore very low. In addition the slow progression of the disease often results in a delay in diagnosis in native British patients, as might, as in this case, the presence of HLA-B27.

Our patient, apart from the synovitis of his left knee and mild backache, was initially fit and well and remained so for many months. The clinical picture and negative $\mathrm{X}$-rays, in conjunction with his HLAB27 positivity, made the initial diagnosis of a seronegative spondarthritis a likely one. On final presentation, he did not complain of any thoracic pain and still only had mild low back ache, but the presence of the cold abscess left little doubt as to the true diagnosis. Direct staining of his knee aspirate for acid-fast bacilli at this time was negative, but culture was positive after 6 weeks.

It is suggested that synovial fluid culture for $M$. tuberculosis is a sensible screening procedure in patients with a seronegative arthritis in whom the diagnosis is not clear cut.

\section{Acknowledgments}

I am grateful to Dr A. Ebringer and Dr M. Shipley for their advice, to Col. M. Brown, Col. B. Simpson and the Ministry of Defence for permission to publish, and to Miss G. Reilly for preparing the typescript.

\section{References}

Brewerton, D.A., Caffrey, M., Nichols, A., Walters, D., OATES, J.K. \& JAMES, D.C.O. (1973a) Reiter's disease and HLAB27. Lancet, ii, 996.

Brewerton, D.A., Hart, F.D., Nichols, A., Caffrey, M., James, D.C.O. \& STURROCK, R.D. (1973b) Ankylosing spondylitis and HLA-B27. Lancet, i, 904.

HALSEY, J.P., ReEBACK, J.S. \& BARNES, C.G. (1982) A decade of skeletal tuberculosis. Annals of Rheumatic Diseases, 41, 7.

LAWRENCE, J.S. (1977) Rheumatism in Populations, p. 283. Heinemann, London.

Newton, P., SharP, J. \& Barnes, K.L. (1982) Bone and joint tuberculosis in Greater Manchester: 1969-79. Annals of Rheumatic Diseases, 41, 1.

(Accepted 4 June 1982) 